\title{
Analgesia Pós-Operatória em Cirurgia Ortopédica: Estudo Comparativo entre o Bloqueio do Plexo Lombar por Via Perivascular Inguinal (3 em 1) com Ropivacaína e a Analgesia Subaracnóidea com Morfina *
}

\author{
Postoperative Analgesia Following Orthopedic Surgery: A Study \\ Comparing Perivascular Lumbar Plexus Inguinal Block with \\ Ropivacaine (3 in 1) and Spinal Anesthesia with Morphine
}

\author{
Neuber Martins Fonseca, TSA $^{1}$, Roberto Araújo Ruzi ${ }^{2}$, Fernando Xavier Ferreira ${ }^{2}$, Fabrício Martins Arruda ${ }^{3}$
}

\begin{abstract}
RESUMO
Fonseca NM, Ruzi RA, Ferreira FX, Arruda FM - Analgesia Pós-Operatória em Cirurgia Ortopédica: Estudo Comparativo entre o Bloqueio do Plexo Lombar por Via Perivascular Inguinal (3 em 1) com Ropivacaína e a Analgesia Subaracnóidea com Morfina
\end{abstract}

Justificativa e Objetivos - O bloqueio do plexo lombar pelo acesso perivascular inguinal, chamado de bloqueio 3 em 1 , tem sido utilizado para analgesia pós-operatória. O objetivo deste estudo foi comparar a analgesia pós-operatória do bloqueio 3 em 1 a da morfina subaracnóidea em pacientes submetidos a cirurgias ortopédicas em membro inferior (MI). cirurgia ortopédica de $\mathrm{MI}$, de ambos os sexos, estado físico ASA I e II, com idades entre 15 e 75 anos, distribuídos em 2 grupos (M e BPL). Foi realizada anestesia subaracnóidea em todos os pacientes, em $L_{3}-L_{4}$ ou $L_{4}-L_{5}$, com $20 \mathrm{mg}$ de bupivacaína isobárica a $0,5 \%$. No grupo $M(n=20)$ foi associado $50 \mu \mathrm{g}$ de morfina ao anestésico local. No grupo BPL $(n=20)$ foi realizado o bloqueio 3 em 1 ao término da cirurgia, utilizando $200 \mathrm{mg}$ de ropivacaína a $0.5 \%$. Avaliou-se a analgesia e a intensidade da dor às 4, 8, 12, 14, 16, 20 e 24 horas após o término da cirurgia, o nível do bloqueio subaracnóideo, o tempo cirúrgico e as complicações.

Resultados - A duração da analgesia no grupo BPL foi de 13,1 $\pm 2,47$, enquanto no grupo $M$ todos os pacientes referiam dor $e$ ausência de bloqueio motor no primeiro instante avaliado (4 horas). Houve falha do bloqueio de um dos 3 nervos em 3 pacientes. A incidência de náusea e prurido foi significativamente maior no grupo $M$. Quanto à retenção

\footnotetext{
* Recebido do (Received from) Serviço de Anestesiologia (CET/SBA) da Universidade Federal de Uberlândia, Uberlândia, MG

1. Professor Adjunto da Disciplina de Anestesiologia, Responsável pelo CET/SBA da Faculdade de Medicina da Universidade Federal de Uberlândia e Membro da Comissão de Normas Técnicas da Sociedade Brasileira de Anestesiologia.

2. Anestesiologista do Serviço de Anestesiologia (SEANE) do Hospital de Clínicas da Faculdade de Medicina da Universidade Federal de Uberlândia

3. $M E_{2}$ do CET/SBA da Faculdade de Medicina da Universidade Federal 3e $M E_{2}$ do $C E T$ Uberlândia.
de
}

Apresentado (Submitted) em 15 de julho de 2002

Aceito (Accepted) para publicação em 06 de setembro de 2002

Correspondência para (Mail to):

Dr. Neuber Martins Fonseca

Rua Antonio Luis Bastos, 300 - Altamira II

38411-116 Uberlândia, MG

E-mail: neuber@triang.com.br

(C) Sociedade Brasileira de Anestesiologia, 2003
Método - Foram estudados 40 pacientes escalados para

urinária, não houve diferença significante entre os grupos. Não houve depressão respiratória, hipotensão arterial ou bradicardia. A analgesia pós-operatória foi mais efetiva no grupo BPL, comparada ao grupo $M$ às $4,8,12,14$ e 16 horas. Às 20 e 24 horas não houve diferença significante entre os grupos.

Conclusões - $A$ analgesia pós-operatória proporcionada pelo bloqueio $3 \mathrm{em} 1$ apresentou efeitos colaterais inferiores à morfina subaracnóidea com tempo de analgesia semelhante.

UNITERMOS: ANALGESIA: Pós-Operatória; ANALGÉSICOS, Opióides: morfina; ANESTÉSICOS, Local: ropivacaína; TÉCNICAS ANESTÉSICAS, Regional: bloqueio 3 em 1 , subaracnóidea

\section{SUMMARY}

Fonseca NM, Ruzi RA, Ferreira FX, Arruda FM - Postoperative Analgesia Following Orthopedic Surgery: A Study Comparing Perivascular Lumbar Plexus Inguinal Block with Ropivacaine (3 in 1) and Spinal Anesthesia with Morphine

Background and Objectives - Perivascular Lumbar plexus inguinal block, (3-in-1 block) has been used for postoperative analgesia. This study aimed at comparing postoperative analgesia of 3-in-1 block and spinal morphine in patients submitted to lower limb orthopedic surgeries (LL).

Methods - Forty ASA I - II patients of both genders, aged 15 to 75 years, scheduled for $L L$ orthopedic surgeries, were distributed in two groups: (M and LPB). Spinal anesthesia was performed in all patients at $L_{3}-L_{4}$ or $L_{4}-L_{5}$ with $20 \mathrm{mg}$ of $0.5 \%$ isobaric bupivacaine. In group $M(n=20), 50 \mu g$ morphine were associated to local anesthetics. In group LPB $(n=20) 3$-in-1 blockade was performed after surgery with $200 \mathrm{mg}$ of $0.5 \%$ ropivacaine. Analgesia and pain intensity were evaluated at 4, 8, 12, 14, 16, 20 and 24 hours after surgery completion, in addition to spinal blockade level, surgery duration and complications.

Results - Analgesia duration in group LPB was $13.1 \pm 2.47$ while all group $M$ patients referred pain and lack of motor block in the first moment evaluated (4 hours). There has been blockade failure in one of the three nerves in three patients. The incidence of nausea and pruritus was significantly higher in group $M$. There was no significant difference between groups in urinary retention. There were no respiratory depression, arterial hypotension or bradycardia. Postoperative analgesia was more effective in group $L P B$ as compared to group $M$ at 4, 8, 12, 14 and 16 hours. There were no significant differences between groups at 20 and 24 hours.

Conclusions - Postoperative analgesia induced by 3-in-1 blockade showed less side-effects as compared to spinal morphine with similar analgesia duration.

KEYWORDS: ANALGESIA: Postoperative, ANALGESICS, Opioids: morphine; ANESTHETICS, Local: ropivacaine; ANESTHETIC TECHNIQUES, Regiona: 3-in-1, spinal block 


\section{INTRODUÇÃO}

C irurgias ortopédicas, especialmente nos membros inferiores, estão associadas com dor pós-operatória de intensidade moderada a intensa, elevando a morbidade dos pacientes $^{1,2}$. Diferentes técnicas e vias são utilizadas com objetivo de reduzir ou abolir este desconforto. A morfina subaracnóidea tem sido efetiva para alívio da dor pós-operatória em alguns tipos de cirurgias ortopédicas ${ }^{3,4}$, porém mostra-se insuficiente para outros ${ }^{5}$, que associada a alguns efeitos colaterais como náuseas, vômitos, retenção urinária e prurido limitam a técnica em alguns pacientes. Diferentemente do bloqueio regional de membro superior, a anestesia do plexo lombar não é feita de rotina por limitações técnicas e falta de treinamento ${ }^{1}$, embora estudos recentes mostrem baixa incidência de efeitos colaterais quando comparados à anestesia espinhal para analgesia pós-operatória de cirurgias ortopédicas no joelho, quadril ou fêmur ${ }^{6-8}$.

A técnica do bloqueio do plexo lombar pelo acesso paravascular inguinal, com objetivo de bloquear os nervos femoral, cutâneo lateral da coxa e obturador, conhecido como bloqueio 3 em 1 , foi descrita em 1973 por Winnie e col. ${ }^{9}$. Os autores sugeriram um mecanismo anatômico no qual imaginaram a existência de um conduto fascial muscular que englobava os nervos femoral $\left(L_{2}, L_{3}, L_{4}\right)$, cutâneo lateral da coxa $\left(L_{2}, L_{3}\right)$ e obturador $\left(L_{2}, L_{3}, L_{4}\right)^{10}$, permitindo que o bloqueio pudesse ser realizado por injeção única de anestésico local, imediatamente abaixo do ligamento inguinal. A dispersão cefálica do anestésico local, associada a compressão distal do local de injeção confirmam esta hipótese ${ }^{11,12}$. A área de anestesia abrangida pelo bloqueio inclui a parte anterior, medial e lateral da coxa, face medial da perna, assim como o periósteo do fêmur e do joelho ${ }^{1,10,13}$. É facilmente realizado, sendo indicado para procedimentos no joelho ${ }^{14-18}$, fêmur ${ }^{19,20}$, quadril $1,21-24$ e biópsia muscular ${ }^{25}$. Tem sido relatado que o bloqueio 3 em 1 proporciona analgesia satisfatória, prolongada, com baixo índice de efeitos colaterais, incluindo alterações hemodinâmicas, freqüentemente potenciais à anestesia peridural ou subaracnóidea ${ }^{21,22}$.

Muitos investigadores têm questionado a eficácia do bloqueio 3 em $1^{26-31}$, enquanto a qualidade da analgesia pós-operatória em cirurgias ortopédicas tem sido exaustivamente confirmada por outros ${ }^{22-24,32}$. O benefício do bloqueio comparado ao uso da morfina subaracnóidea tem sido pouco investigado e com resultados insatisfatórios ${ }^{2}$.

O objetivo deste estudo foi avaliar a analgesia pós-operatória do bloqueio 3 em 1 com ropivacaína a $0,75 \%$ comparativamente à morfina por via subaracnóidea em pacientes submetidos a cirurgias ortopédicas de fêmur, quadril ou joelho.

\section{MÉTODO}

Após a aprovação do método pelo Comitê de Ética Médica do Hospital de Clínicas da Universidade Federal de Uberlândia e do consentimento por escrito, foram estudados 40 pacientes, de ambos os sexos, classificados quanto ao estado físi- co em ASA I ou II, com idades entre 15 e 75 anos, escalados para cirurgias ortopédicas de fêmur, quadril ou joelho, distribuídos aleatoriamente por sorteio, em 2 grupos de igual número. Foram excluídos os pacientes que apresentavam distúrbios de coagulação, infecção no local da punção, sinais clínicos de hipovolemia, história de alergia a anestésicos locais, aqueles que não pudessem colaborar na avaliação pós-operatória, os com trauma ou dor em outro local do corpo e usuários de drogas.

No centro cirúrgico, foi estabelecida hidratação venosa pela inserção de cateter $16 \mathrm{G}$ ou $18 \mathrm{G}$ em veia do antebraço, cerca de 30 minutos antes de encaminhar o paciente à sala de cirurgia, e iniciada a infusão de solução de Ringer com lactato. Esta via foi utilizada para medicação pré-anestésica com 1 a $3 \mathrm{mg}$ de midazolam. Na sala de cirurgia, a monitorização constou de medida automática da pressão arterial a cada 3 minutos, cardioscopia na derivação CM5 e oximetria de pulso para análise contínua da saturação periférica de oxigênio $\left(\mathrm{SpO}_{2}\right)$.

Todos os pacientes foram submetidos à anestesia subaracnóidea lombar simples em decúbito lateral, no espaço $L_{3}-L_{4}$ ou $L_{4}-L_{5}$ porvia mediana, utilizando agulha tipo Quincke $25 \mathrm{G}$. Após saída do líquor cefalorraquidiano foram administrados $20 \mathrm{mg}$ de bupivacaína isobárica a $0,5 \%$. Os 40 pacientes foram distribuídos em 2 grupos de igual número para avaliação da analgesia pós-operatória, sendo que em 20 pacientes (grupo M) foi associado $50 \mu \mathrm{g}$ de morfina ao anestésico local no momento da anestesia subaracnóidea e nos outros 20 (grupo BPL) foi feito bloqueio 3 em 1 ao término da cirurgia. Tenoxicam (20 mg) e dipirona $(1 \mathrm{~g})$, por via venosa, foram utilizados em todos os pacientes antes da incisão cirúrgica. Os pacientes do grupo BPL foram colocados na posição supina ao final do procedimento cirúrgico e, após demarcada a anatomia de superfície e anti-sepsia com álcool iodado a $0,25 \%$, realizou-se anestesia infiltrativa com lidocaína a $1 \%$ sem vasoconstritor $(20 \mathrm{mg})$ no local da punção programada para o bloqueio 3 em 1. Com a localização do pulso da artéria femoral ao nível do ligamento inguinal, introduziu-se agulha $22 \mathrm{G}$ de bisel curto de $5 \mathrm{~cm}$ de comprimento e eletricamente isolada por teflon, conectada a estimulador de nervo periféri$\mathrm{co}$, introduzida com ângulo de $60^{\circ} \mathrm{em}$ relação à pele, $1 \mathrm{~cm}$ lateralmente ao pulso arterial e $2 \mathrm{~cm}$ abaixo do ligamento inguinal. A agulha foi introduzida gradualmente com objetivo de estimular o nervo femoral. O estímulo elétrico aplicado foi inicialmente ajustado com freqüência de $1 \mathrm{~Hz}$ e corrente de 1,0 $\mathrm{mA}$. Como a contração muscular do quadríceps representava resposta motora ao estímulo elétrico, a corrente foi reduzida a 0,5 mA. Caso o movimento parasse, a posição da aguIha era reajustada para manter resposta muscular adequada com baixa corrente $(<0,5 \mathrm{~mA})$, considerado ponto ideal para a injeção do anestésico local. Não foi pesquisada parestesia. Após dose teste com $2 \mathrm{ml}$ da solução anestésica, para verificar a possibilidade de injeção anestésica intraneural (dor aguda concomitante à injeção anestésica), seguiu-se injeção lenta da dose total de $200 \mathrm{mg}$ de ropivacaína a 0,5\%, interrompida a cada $5 \mathrm{ml}$ e aspiração do êmbolo da seringa 
para confirmar que a agulha encontrava-se em região extravascular.

Todos os bloqueios foram realizados e avaliados por anestesiologista com experiência em anestesia regional, sendo investigada a analgesia pós-operatória pelo teste de picada da agulha para determinar a extensão dos bloqueio sensitivo para os nervos femoral, lateral cutâneo da coxa e obturador. A graduação da dor foi avaliada pela escala analógica visual (EAV) de dor de 0 a 10, considerando " 0 " como sem dor e"10" como dor insuportável ${ }^{33,34}$, nos momento de 4, 8, 12, 14,16, 20, 24 horas, após o término da cirurgia.

A duração dos bloqueios motor e sensorial foi definido como o tempo entre a injeção do anestésico e a recuperação das funções motora e sensorial, respectivamente.

Os pacientes foram estudados durante 24 horas para verificação de complicações no local da punção. Diclofenaco (75 $\mathrm{mg}$ ) por via muscular foi prescrito aos pacientes que referissem dor pela EAV superior ou igual a 4. Foram avaliados complicações e efeitos adversos como presença de náuseas pela escala analógica visual (EAV) proposta por Boogaerts e col. ${ }^{35}$ para avaliar objetivamente a intensidade de náuseas e vômitos no pós-operatório, hipotensão arterial (diminuição de $20 \%$ da pressão arterial média), bradicardia (batimentos inferiores a $50 \mathrm{bpm}$ ), depressão respiratória (freqüência respiratória menor que 8 irpm), prurido ou retenção urinária.

O estudo estatístico foi feito pelo teste $t$ de Student com objetivo de estudar as características gerais dos pacientes quanto ao peso, idade, altura, duração da cirurgia e para comparar a analgesia pós-operatória do grupo M com o grupo BPL nos momentos estudados. Foi utilizada a análise de variância por postos de Friedman para avaliar a analgesia pós-operatória no grupo e complementado pelo teste de comparações múltiplas quando apresenta-se diferença significante. Sexo, estado físico, prurido, retenção urinária, náuseas e vômitos (EAV maior ou igual a 4) foram estudados pelo teste do Qui-quadrado $\left(x^{2}\right)$. As medidas relativas à altura do bloqueio dispensaram análise, porque os dados são apresentados em escala nominal. Em todos os testes, fixou-se em $5 \%$ o nível para rejeição da hipótese de nulidade, assinalando-se com asterisco os valores significantes.

\section{RESULTADOS}

Os grupos foram semelhantes quanto aos dados antropométricos, tempo cirúrgico, tipo de cirurgia realizada e nível de bloqueio subaracnóideo (Tabelas I, II e III).

Em todos os pacientes foi possível a localização do nervo femoral com auxílio do estimulador de nervo periférico. O sucesso do bloqueio 3 em 1 (bloqueio sensitivo dos nervos femoral, cutâneo lateral da coxa e obturatório) foi de $85 \%$, ou seja, houve falha (falha da anestesia de um dos 3 nervos) em 3 dos pacientes. O sucesso da anestesia foi de 100\% (20 pacientes) para bloqueio do nervo femoral, $90 \%$ (18 pacientes) para o nervo obturatório e $85 \%$ (17 pacientes) para o nervo cutâneo lateral da coxa, conforme mostra a figura 1.
Tabela I - Dados Demográficos

\begin{tabular}{ccc}
\hline & Grupo BPL $(\mathrm{n}=20)$ & Grupo M $(\mathrm{N}=20)$ \\
\hline Idade (anos) $^{*}$ & $44,1 \pm 20,1$ & $39,2 \pm 14,6$ \\
Limites & 15 a 75 & 15 a 64 \\
Peso $(\mathrm{kg})^{*}$ & $70,4 \pm 18,1$ & $70,1 \pm 11,3$ \\
Limites & 55 a 100 & 50 a 94 \\
Altura (cm) ${ }^{*}$ & $168 \pm 9,3$ & $164 \pm 6,1$ \\
Limites & 160 a 185 & 157 a 180 \\
Estado Físico & & \\
ASA I & 15 & 16 \\
ASA II & 5 & 4 \\
Sexo & 13 & 12 \\
Masculino & 7 & 8 \\
Feminino & & \\
\hline
\end{tabular}

* Valores expressos pela Média \pm DP

Tabela II - Tempo de Cirurgia (Média \pm DP) e Altura do Bloqueio Anestésico

\begin{tabular}{lcc}
\hline & Grupo BPL $(n=20)$ & Grupo M $(n=20)$ \\
\hline Nível de bloqueio & T10 & T8 \\
Limites & T6 a T12 & T6 a T10 \\
Tempo cirúrgico $(\mathrm{min})$ & $137 \pm 50,0$ & $126 \pm 44,2$ \\
Limites & 30 a 210 & 30 a 180 \\
\hline
\end{tabular}

Tabela III - Cirurgias Realizadas

\begin{tabular}{lcc}
\hline Cirurgias & Grupo BPL $(n=20)$ & Grupo M $(n=20)$ \\
\hline Fêmur & 9 & 13 \\
Joelho & 5 & 4 \\
Quadril & 6 & 3 \\
\hline
\end{tabular}

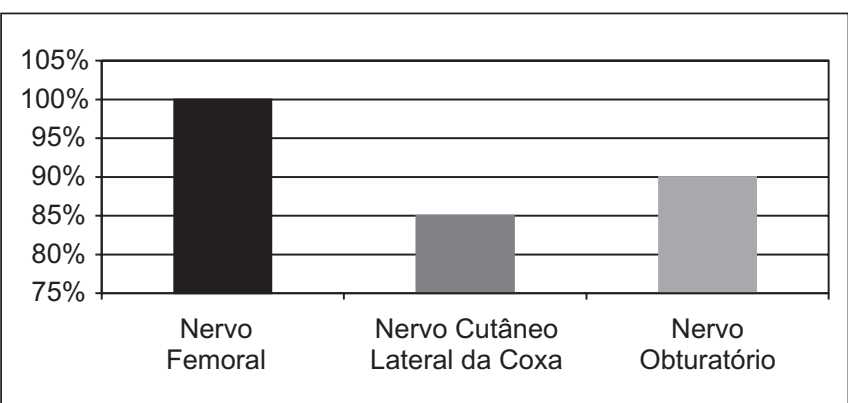

Figura 1 - Índice de Sucesso (\%) dos Nervos Femoral, Cutâneo Lateral da Coxa e Obturatório no Bloqueio 3 em 1

A duração do bloqueio motor no grupo BPL foi de 9,9 $\pm 3,54$ horas e da analgesia (teste da picada de agulha) de 13,1 \pm 2,47 horas variando de 12 a 16 horas. No grupo M os pacientes referiam dor à picada de agulha e ausência de bloqueio motor no primeiro instante observado (4 horas).

Revista Brasileira de Anestesiologia Vol. 53, № 2, Março - Abril, 2003 
Observou-se incidência significantemente menor de náuseas, acima de 4 pela Escala Analógica Visual (EAV), e de prurido no grupo BPL em relação ao grupo $\mathrm{M}$, conforme mostram respectivamente as figuras 2 e 3 . Retenção urinária ocorreu em 1 paciente do grupo BPLe 5 pacientes do grupo M; porém, sem diferença significante entre os grupos. Não foram observadas depressão respiratória, hipotensão arterial e bradicardia, nos momentos estudados nos grupos.

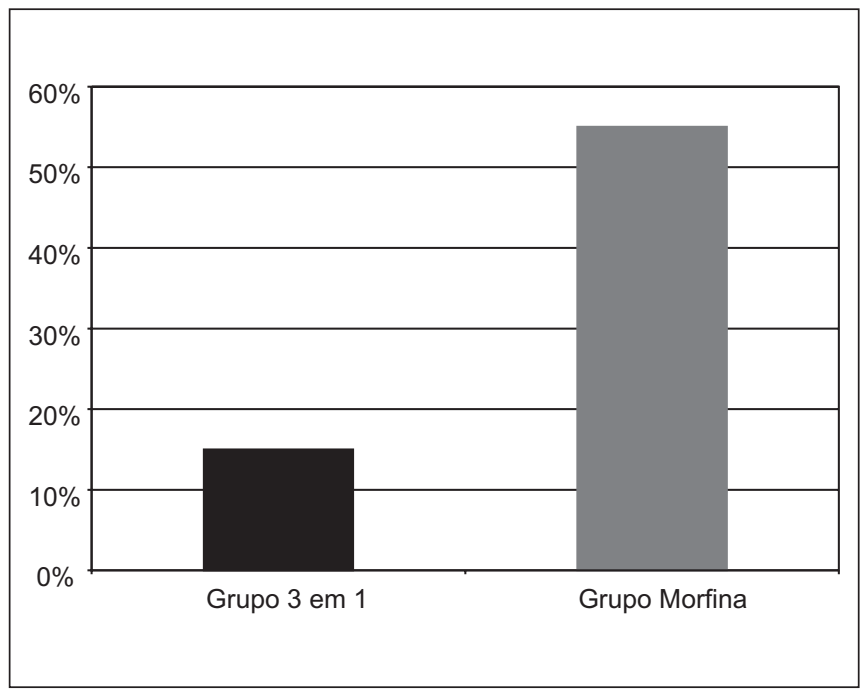

Figura 2 - Incidência em Percentagem de Náusea maior ou igual a 4 pela EAV nos Grupos Estudados

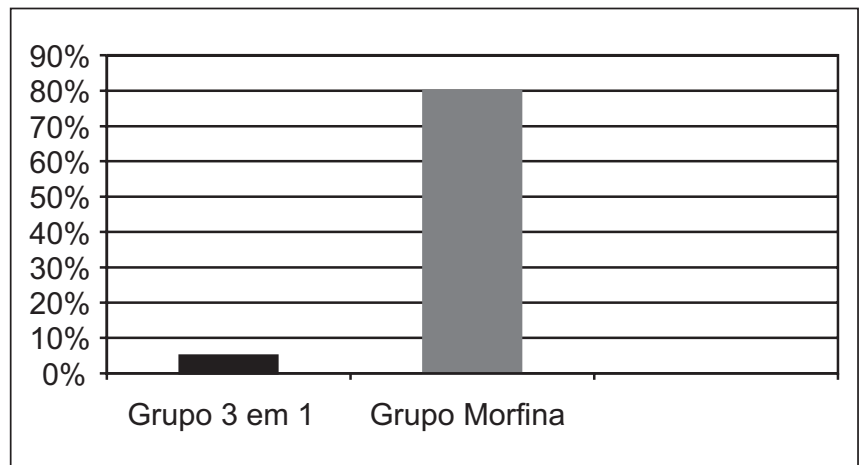

Figura 3 - Incidência em Percentagem de Prurido nos Grupos Estudados

AEAV de dor mostrou menores índices no grupo BPL comparada ao grupo $M$ às $4,8,12,14$ e 16 horas $(p<0,01)$. Às 20 e 24 horas não houve diferença significante. A análise entre os momentos do grupo M não mostrou diferença significante, diferentemente do grupo BPL, que mostrou diferença significante até o momento 16 horas (Figura 4).

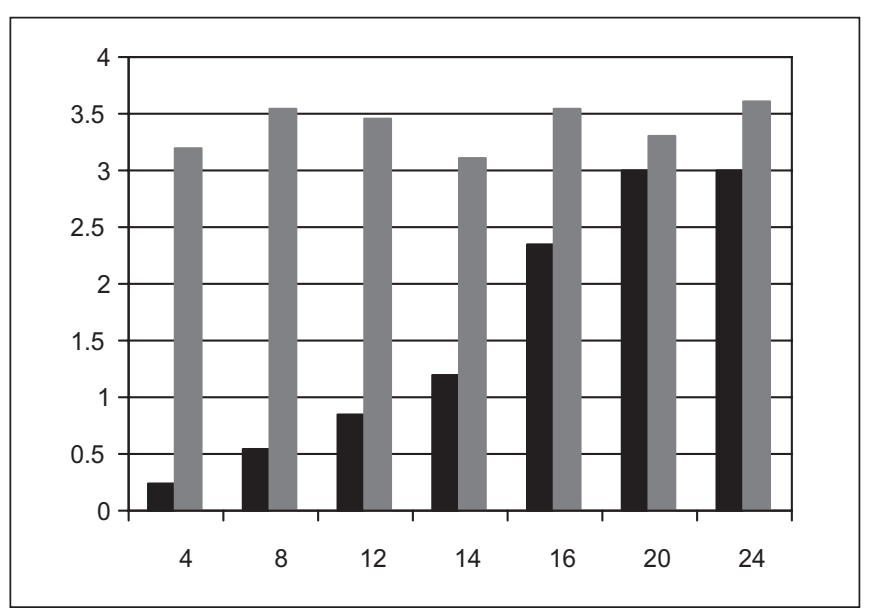

Figura 4 - Escala Analógica Visual ( 0 a 10) de Dor nos Grupos Estudados durante os Momentos de 4, 8, 12, 14, 16, 20 e 24 horas

A necessidade de analgésicos foi de $60 \%$ no grupo BPL e de $75 \%$ no grupo $\mathrm{M}$. Em todos os pacientes, a cirurgia foi conduzida sem necessidade de anestesia complementar. Nenhum paciente necessitou de cateter vesical no pós-operatório ou permaneceu com ele.

\section{DISCUSSÃO}

O estudo mostrou que a morfina subaracnóidea e o bloqueio do plexo lombar pela técnica 3 em 1 foram efetivos para analgesia pós-operatória nos pacientes submetidos a cirurgias ortopédicas de quadril, fêmur ou joelho. O limiar analgésico da morfina, apesar de mostrar menor efetividade, apresentou efeito pós-operatório analgésico mais constante que o bloqueio femoral. A associação de morfina subaracnóidea ao anestésico previamente ao bloqueio subaracnóideo é técnica simples e de fácil execução, sistematicamente consagrada para analgesia pós-operatória de cirurgias traumato-ortopédicas dos membros inferiores. Após o bloqueio subaracnóideo, a morfina atua no sistema nervoso central dentro de 60 minutos $^{36}$. Popularizou-se pela facilidade de administração, porém alguns estudos falham em demonstrar benefícios com a técnica, particularmente relacionados aos efeitos colaterais relativamente comuns como sedação, náuseas e prurido, especialmente com altas doses de morfina subaracnóidea ${ }^{6,7}$.

O bloqueio do plexo lombar pela técnica 3 em 1 é proposto para analgesia pós-operatória de cirurgias de joelho 6,16,18,29,37-39 , osteossíntese de fêmur ${ }^{19}$ e cirurgias de quadril ${ }^{24}$, sendo rotina em alguns serviços ${ }^{6}$.

Estudo com distribuição aleatória, examinando a infusão contínua de anestésico local no nervo femoral, demonstrou melhor analgesia, comparada ao uso sistêmico de opióides 6,39 . Outros estudos não mostraram diferença analgésica com dose simples (única) ou infusão contínua para bloqueio do nervo femoral ${ }^{17,38}$. Analgesia inconsistente ao bloqueio do nervo femoral pode ser explicada pela persistência da sensação da região posterior da perna, indicando que o ner- 
vo ciático deve estar relacionado à sensação dolorosa da perna $^{42}$. Bloqueio combinado lombar e ciático proposto por Misra e col. ${ }^{43}$ sugere vantagens em relação ao bloqueio isolado, porém a dose de bupivacaína utilizada de $3 \mathrm{mg} . \mathrm{kg}^{-1}$, necessária à técnica, pode exceder a recomendada, sendo potencialmente tóxica. Contudo, apesar da dose, se o nervo ciático fosse bloqueado simultaneamente ao bloqueio femoral, ocorreria perda da simplicidade da técnica com aumento dos riscos, não justificando portanto os benefícios.

O tempo de analgesia observado com o bloqueio $3 \mathrm{em} 1$ neste estudo $(13,1 \pm 2,47$ horas) corrobora o observado por outros autores, em que a analgesia foi de 15 horas com bupivacaína a $0,25 \%{ }^{1}, 14$ a 23 horas com bupivacaína a 0,25\% $41,44,45$ e de 11 e 13 horas com ropivacaína a $0,75 \%$ e a $0,5 \%$ respectivamente ${ }^{44-47}$.

A técnica clássica de Winnie, proposta para o bloqueio 3 em 1 , utiliza $20 \mathrm{ml}$ de anestésico. O presente estudo optou por volume maior visando diminuir a incidência de falhas, como sugerido por outros autores ${ }^{19,20}$. A falha observada no presente estudo foi semelhante a estudos prévios em que ocorreu de $7 \%$ a $20 \%{ }^{1,41,45,46}$

Diversos anestésicos podem ser utilizados para o bloqueio 3 em 1 , como prilocaína a $0,75 \%{ }^{11}$, mepivacaína a $2 \%{ }^{44}$, lidocaína a $1 \%{ }^{11}$, clorprocaína a $3 \%{ }^{11}$, bupivacaína a $0,25 \%$ e $0,5 \%{ }^{16,23,40,41,44,45}$ e ropivacaína a $0,5 \%$ ou a $0,75 \%{ }^{44,45,47}$. Optou-se pela ropivacaína, neste estudo, pelas características farmacológicas do agente, como longa duração e baixa cardiotoxicidade ${ }^{46,48}$.

Diferentes métodos são descritos para o bloqueio 3 em 1 , como pesquisa de parestesias ${ }^{5}$, perda de resistência ${ }^{17}$ ou com a auxílio de estimulador de nervos periféricos $1,19,37,41,49,50$. O estimulador de nervos periféricos tem sido a técnica de eleição para localização de nervos por não promover lesão mecânica, respeitados os limites de amperagem, voltagem e aplicação em nervo misto ${ }^{51}$, sendo os adotados no presente estudo.

O bloqueio motor prolongado nos pacientes do grupo 3 em 1 $(9,9 \pm 3,54$ horas $)$ foi fator limitante à técnica. Sugere-se realização de outros estudos com redução da concentração anestésica para confirmar o benefício analgésico da técnica com redução do bloqueio motor.

Avantagem do bloqueio do plexo lombar pela técnica do 3 em 1 é a baixa incidência de complicações. Há, contudo, citações de neuropatia femoral ${ }^{52}$ e síndrome compressiva do nervo femoral por hematoma ${ }^{53}$. Outras complicações referidas são punção arterial, injeção intravascular de anestésico local, anestesia peridural, infecção no local de punção, traumatismo do nervo, cruralgia regressiva ${ }^{54-56}$. A baixa incidência de complicações pode ser explicada por reduzida absorção sistêmica de anestésico local ${ }^{37}$. Este estudo corrobora estas observações por não apresentar complicações nos pacientes estudados.

Atécnica de bloqueio 3 em 1 é de fácil execução, baixo risco e curta latência para alívio doloroso. As observações deste estudo apresentam boa relação risco/benefício entre as técnicas de anestesia locorregional, superior à morfina subarac- nóidea para analgesia pós-operatória em pacientes submetidos aos procedimentos realizados neste estudo.

A necessidade de analgésico no pós-operatório foi de $60 \%$ no grupo BPL e $75 \%$ no grupo M, diferentemente do estudo de Edkin e col. ${ }^{57}$ em que $92 \%$ do pacientes não necessitaram de analgésicos nas primeiras 24 horas do pós-operatório. Esta diferença provavelmente foi devida ao fato de que a administração do analgésico utilizado no pós-operatório foi baseada na EAV, adotando-se a administração sempre que a escala mostrava-se igual ou superior a 4, visando o conforto do paciente, e não por solicitação espontânea do paciente.

A morfina subaracnóidea apresenta maior incidência de efeitos colaterais, como náuseas, vômitos e prurido ${ }^{58}$, corroborando o observado neste estudo. Aausência destes efeitos no grupo com bloqueio 3 em 1 sugere importante vantagem para analgesia pós-operatória dos pacientes estudados, somada à facilidade técnica para execução do bloqueio na posição supina, com conforto aos pacientes que apresentem limitações à manipulação, como aqueles com tração transesquelética, além de objetivar analgesia específica no local da agressão cirúrgica ou do comprometimento doloroso afetado.

\section{Postoperative Analgesia Following Orthopedic Surgery: A Study Comparing Perivascular Lumbar Plexus Inguinal Block with Ropivacaine (3 in 1) and Spinal Anesthesia with Morphine}

Neuber Martins Fonseca, TSA, M.D., Roberto Araújo Ruzi, M.D., Fernando Xavier Ferreira, M.D., Fabrício Martins Arruda, M.D.

\section{INTRODUCTION}

Orthopedic surgeries, especially in lower limbs, are associated to moderate to severe postoperative pain and increased patients' morbidity ${ }^{1,2}$. Different techniques and routes for drugs administration are used aiming at decreasing or abolishing this discomfort. Spinal morphine has been effective for postoperative pain relief in some orthopedic surgeries ${ }^{3,4}$, but is inadequate for others ${ }^{5}$. This fact, associated to some side-effects, such as nausea, vomiting, urinary retention and pruritus, counterindicates the technique for some patients. Differently from upper limb regional block, lumbar plexus anesthesia is not routinely employed due to technical limitations and lack of training ${ }^{1}$, although recent studies have shown a low incidence of side-effects as compared to spinal anesthesia for postoperative pain relief of knee, hip or femur orthopedic surgeries $^{6-8}$.

Paravascular inguinal lumbar plexus block aiming at blocking femoral, lateral cutaneous of thigh and obturator nerves, also known as 3-in-1 blockade, was firstly described in 1973 by Winnie et al. ${ }^{9}$. The authors suggested an anatomical Vol. 53, N 2, Março - Abril, 2003 
mechanism in which they imagined the existence of a fascial muscle conduit encompassing femoral $\left(\mathrm{L}_{2}, \mathrm{~L}_{3}, \mathrm{~L}_{4}\right)$, lateral cutaneous of thigh $\left(\mathrm{L}_{2}, \mathrm{~L}_{3}\right)$ and obturator $\left(\mathrm{L}_{2}, \mathrm{~L}_{3}, \mathrm{~L}_{4}\right)$ nerves ${ }^{10}$, allowing the blockade to be performed by single local anesthetic injection immediately below the inguinal ligament. Local anesthetic cephalad spread associated to distal compression of the injection site confirm such hypothesis ${ }^{11,12}$. Blocked area includes anterior, medial and lateral thigh, medial face of leg, as well as femoral and knee periostium ${ }^{1,10,13}$. It is easily performed, being indicated for knee ${ }^{14-18}$, femur ${ }^{19,20}$, hip ${ }^{1,21-24}$ and muscle biopsy ${ }^{25}$ procedures. It has been reported that the 3-in-1 blockade provides satisfactory and prolonged analgesia with low incidence of side-effects, including hemodynamic changes, which are frequent in epidural or spinal anesthesia ${ }^{21,22}$.

Several investigators have questioned the efficacy of the 3-in-1 blockade ${ }^{26-31}$, while the quality of postoperative analgesia for orthopedic surgeries has been exhaustively confirmed by others ${ }^{22-24,32}$. The benefit of this blockade as compared to spinal morphine has been poorly investigated with unsatisfactory results ${ }^{2}$.

The aim of this study was to evaluate postoperative analgesia of 3 -in- 1 blockade with $0.75 \%$ ropivacaine as compared to spinal morphine in patients submitted to orthopedic femur, hip or knee surgeries.

\section{METHODS}

After the Hospital das Clinicas, Universidade Federal, Uberlandia Ethical Committee approval and their written consent, 40 patients of both genders, aged 15 to 75 years, physical status ASA I or II, scheduled for orthopedic femur, hip or knee surgeries were included in the study. Patients were randomly distributed in two equal groups. Exclusion criteria were coagulation disorders, puncture site infection, clinical signs of hypovolemia, history of allergy to local anesthetics, patients who could not cooperate in postoperative evaluation, trauma patients or those with pains in different body sites, and drug abuse.

An $18 \mathrm{G}$ or $16 \mathrm{G}$ catheter was inserted in the operating center in a forearm vein for hydration with lactated Ringer's, approximately 30 minutes before referring patients to the operating room. This route was used for preanesthetic medication with 1 to $3 \mathrm{mg}$ midazolam. Monitoring in the operating room consisted of automatic blood pressure measurement at 3-minute intervals, cardioscopy at CM5 lead and pulse oximetry for continuous peripheral oxygen saturation analysis $\left(\mathrm{SpO}_{2}\right)$. All patients were submitted to simple lumbar spinal anesthesia in the lateral position in $L_{3}-L_{4}$ or $L_{4}-L_{5}$ with a $25 G$ Quincke needle. After obtaining CSF, $20 \mathrm{mg}$ of $0.5 \%$ isobaric bupivacaine were administered. Patients were distributed in two equal groups for postoperative analgesia evaluation. In group M (20 patients), $50 \mu \mathrm{g}$ morphine were added to local anesthetic solution used in the spinal anesthesia and in group LPB (20 patients), 3-in-1 blockade was performed at surgery completion.
Intravenous tenoxicam $(20 \mathrm{mg})$ and dipirone $(1 \mathrm{~g})$ were administered to all patients before surgical incision.

Group LPB patients were placed in the supine position at surgery completion and, after marking surface anatomy and cleaning with $0.25 \%$ iodized alcohol, infiltrative anesthesia was performed with $1 \%$ plain lidocaine $(20 \mathrm{mg})$ at the 3 -in-1 blockade puncture site. With the femoral artery pulse location at inguinal ligament level, a $5 \mathrm{~cm}$ short bevel $22 \mathrm{G}$ needle electrically isolated with teflon and connected to a peripheral nerve stimulator was introduced at a $60^{\circ}$ angle to the skin, 1 $\mathrm{cm}$ laterally to arterial pulse and $2 \mathrm{~cm}$ below inguinal ligament. Needle was gradually introduced seeking for femoral nerve stimulation. Electric stimulation was initially adjusted to $1 \mathrm{~Hz}$ frequency and $1.0 \mathrm{~mA}$ current. Obtaining quadriceps contraction response to electric stimulation, current was decreased to $0.5 \mathrm{~mA}$. If the movement stopped, needle position was readjusted to maintain adequate muscle response with low current $(<0.5 \mathrm{~mA})$, and this was considered the optimal site for local anesthetic injection. Paresthesia was not investigated. After a test dose with $2 \mathrm{ml}$ anesthetic solution to check the possibility of intraneural anesthetic injection (acute pain at anesthetic injection), $200 \mathrm{mg}$ of $0.5 \%$ ropivacaine were injected at slow rate and interrupted at every $5 \mathrm{ml}$ for syringe aspiration to confirm that the needle was not inside a vessel.

All blockades were performed and evaluated by anesthesiologists skilled in regional anesthesia. Postoperative analgesia was investigated by the needle prick test to determine the extension of femoral, lateral cutaneous of thigh and obturator nerves sensory block. Pain intensity was evaluated by the visual analog scale (VAS) from 0 to 10 , considering "0" no pain and " 10 " unbearable pain ${ }^{33,34}$, in moments 4, 8, 12, 14, 16, 20 and 24 hours after surgery completion.

Motor and sensory block duration was defined as time between anesthetic injection and motor and sensory function recovery, respectively.

Patients were evaluated for 24 hours for complications at puncture site. Muscular diclofenac $(75 \mathrm{mg})$ was prescribed for patients referring VAS pain 4 or above. Complications and adverse effects such as nausea (assessed by the visual analog scale proposed by Boogaerts et al. ${ }^{35}$ ), arterial hypotension ( $20 \%$ decrease of mean blood pressure), bradycardia (beats below $50 \mathrm{bpm}$ ), respiratory depression (respiratory rate lower than 8 irpm), pruritus and urinary retention were evaluated.

Student's $t$ test was used to evaluate general characteristics such as age, weight, height and surgery duration, as well as to compare postoperative analgesia between groups in the moments studied. Friedman's analysis of variance by posts was used to evaluate postoperative analgesia within each group, and multiple comparisons test was applied in the presence of significant differences. Chi-square test was used to evaluated gender, physical status, pruritus, urinary retention, nausea and vomiting (VAS 4 or above). Blockade intensity was not analyzed because data were presented in nominal scale. For all tests, $5 \%$ was the level determined for null 
hypothesis rejection and significant values were marked with a star $(*)$.

\section{RESULTS}

Groups were similar in demographics data, surgery duration, type of surgery and spinal blockade level (Tables I, II and III).

Table I - Demographics Data

\begin{tabular}{ccc}
\hline & Group LPB $(\mathrm{n}=20)$ & Group M $(\mathrm{N}=20)$ \\
\hline Age (years) * & $44.1 \pm 20.1$ & $39.2 \pm 14.6$ \\
Limits & 15 to 75 & 15 to 64 \\
Weight $(\mathrm{kg})^{*}$ & $70.4 \pm 18.1$ & $70.1 \pm 11.3$ \\
Limits & 55 to 100 & 50 to 94 \\
Height $(\mathrm{cm}){ }^{*}$ & $168 \pm 9.3$ & $164 \pm 6.1$ \\
Limits & 160 to 185 & 157 to 180 \\
Physical status & & 16 \\
ASA I & 15 & 4 \\
ASA II & 5 & 12 \\
Gender & 13 & 8 \\
Male & 7 & \\
Female & & \\
\hline
\end{tabular}

*Values expressed in Mean \pm SD

Table II - Surgery Duration (Mean \pm SD) and Anesthetic Blockade Level

\begin{tabular}{lcc}
\hline & Group LPB $(n=20)$ & Group M $(n=20)$ \\
\hline Blockade level & T10 & T8 \\
Limits & T6 to T12 & T6 to T10 \\
Surgery duration (min) & $137 \pm 50,0$ & $126 \pm 44,2$ \\
$\quad$ Limits & 30 to 210 & 30 to 180 \\
\hline
\end{tabular}

Table III - Types of Surgeries

\begin{tabular}{lcc}
\hline Surgeries & Group LPB $(n=20)$ & Group M $(n=20)$ \\
\hline Femur & 9 & 13 \\
Knee & 5 & 4 \\
Hip & 6 & 3 \\
\hline
\end{tabular}

The femoral nerve could be located in all patients with the aid of the peripheral nerve stimulator. The success of the 3-in-1 blockade (femoral, lateral cutaneous of thigh and obturator nerves sensory block) was $85 \%$, that is, in 3 patients, there was at least one nerve block failure. Anesthesia success was $100 \%$ (20 patients) for femoral nerve, 90\% (18 patients) for obturator nerve and $85 \%$ (17 patients) for lateral cutaneous of thigh, as shown in figure 1.

Motor block duration in group LPBwas $9.9 \pm 3.54$ hours and analgesia duration (needle prick test) was $13.1 \pm 2.47$ hours, varying from 12 to 16 hours. Group M patients referred pain at needle prick and lack of motor block in the first moment observed (4 hours).

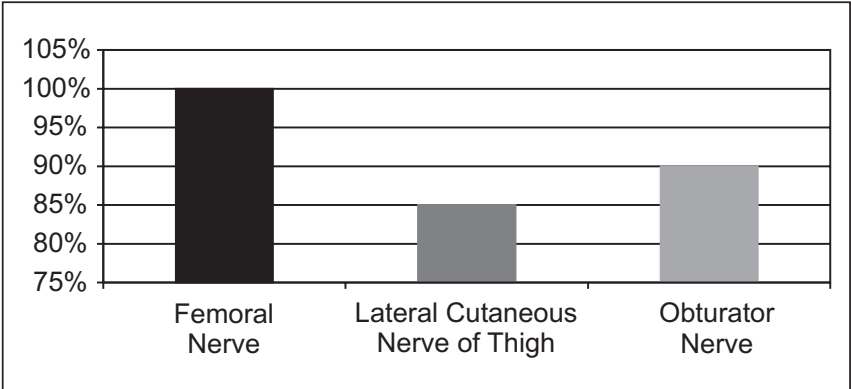

Figure 1 - Success Rate (\%) of 3-in-1 Blockade of Femoral, Lateral Cutaneous of Thigh and Obturator Nerves

There was a significant lower incidence of nausea (VAS > 4) and pruritus in group LPB as compared to group $M$, as shown in figures 2 and 3 , respectively. One group LPB patient had urinary retention against 5 in group $M$, however with no significant difference between groups. There were no respiratory depression, arterial hypotension or bradycardia in all moments studied.

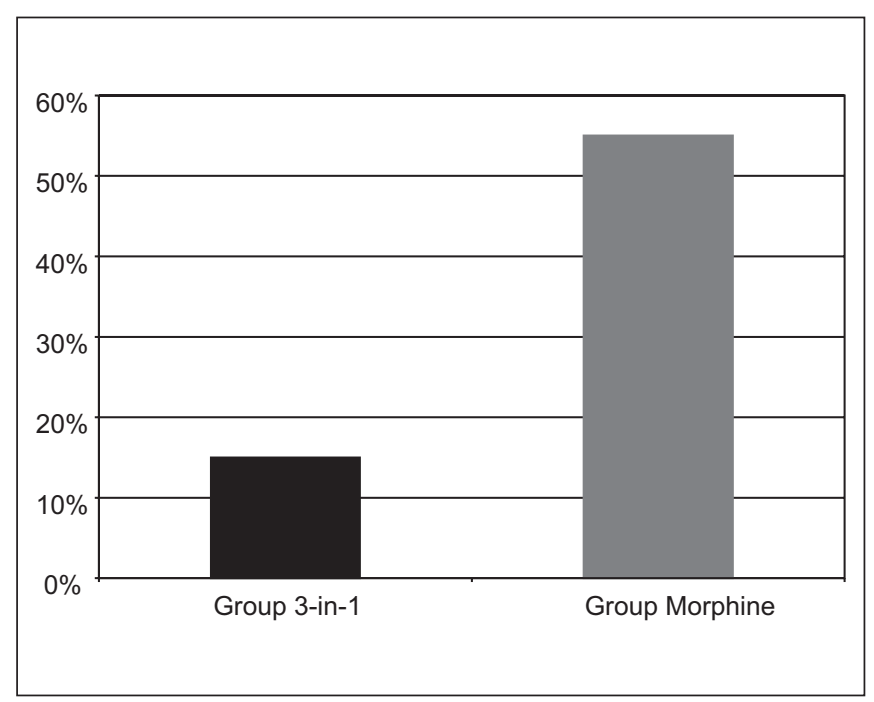

Figure 2 - Incidence of VAS 4 or above Nausea (\%)

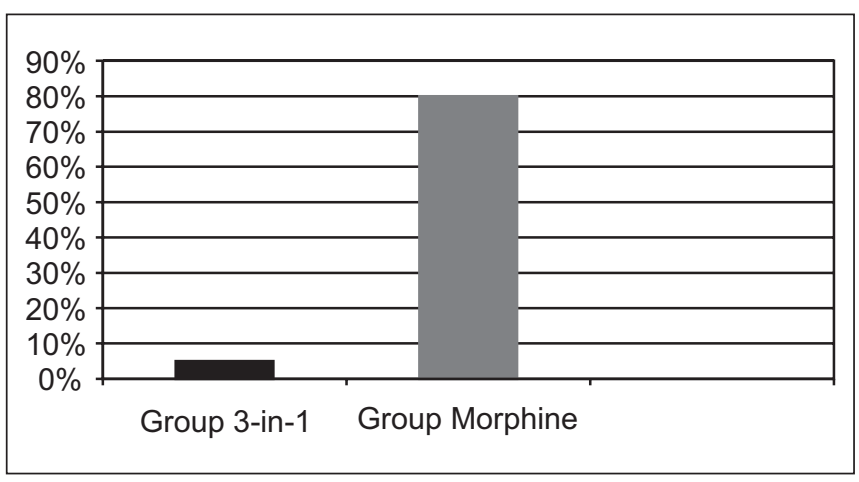

Figure 3 - Incidence of Pruritus (\%)

Revista Brasileira de Anestesiologia Vol. 53, № 2, Março - Abril, 2003 
Pain VAS showed lower scores in group LPB as compared to group $M$ at 4, 8, 12, 14 and 16 postoperative hours $(p<0.01)$. There were no significant differences at 20 and 24 hours. There were no significant differences among moments in group $M$, differently from group LPB which showed significant differences until 16 hours (Figure 4).

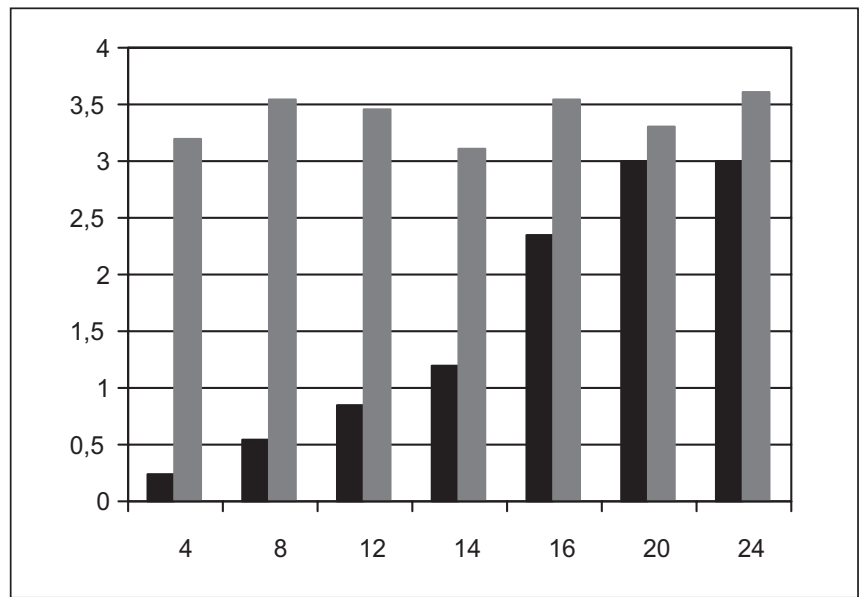

Figure 4 - Pain Visual Analog Scale (0 to 10) in Both Groups during Moments 4, 8, 12, 14, 16, 20 and 24 hours

Analgesics were needed in $60 \%$ of group LPB and $75 \%$ of group M patients. Surgery was performed in all patients never requiring any anesthetic complementation. No patient needed postoperative vesical catheter or remained with it.

\section{DISCUSSION}

Our study has shown that spinal morphine and lumbar plexus blockade by the 3 -in- 1 technique were effective for postoperative analgesia in patients submitted to orthopedic hip, femur or knee surgeries. Although being less effective spinal morphine had a more constant postoperative analgesic effect as compared to femoral block. The addition of spinal morphine to the anesthetic solution before spinal block is a simple, easy and widely accepted way to provide postoperative analgesia for lower limb traumato-orthopedic surgeries. After spinal block, morphine acts on the central nervous system within 60 minutes ${ }^{36}$. It became popular for its easy administration, but some studies have failed in showing benefits with the technique, especially regarding the relatively common side-effects, such as sedation, nausea and pruritus, frequently seen with high spinal morphine doses ${ }^{6,7}$.

Lumbar plexus block by the 3 -in-1 technique is proposed for postoperative analgesia in knee 6,16,18,29,37-39, femur osteosynthesis ${ }^{19}$ and hip surgeries ${ }^{24}$, being a routine in some hospitals ${ }^{6}$.

A randomized study evaluating local anesthetics continuous infusion in the femoral nerve has shown better analgesia as compared to systemic opioids ${ }^{6,39}$. Other studies have not shown analgesic differences with a single dose or continuous infusion for femoral nerve blockade ${ }^{17,38}$. Inconsistent analgesia with femoral nerve blockade may be associated to the persistence of sensation in the posterior region of the leg, indicating that the sciatic nerve may be related to painful leg sensation ${ }^{42}$. The combined lumbar and sciatic block proposed by Misra et al. ${ }^{43}$ suggests advantages as compared to isolated block, but the $3 \mathrm{mg} . \mathrm{kg}^{-1}$ bupivacaine dose needed for the technique may exceed the recommended dose, thus being potentially toxic. However, and regardless of the dose, if the sciatic nerve is to be blocked simultaneously to femoral block, the technique will lose simplicity with increased risks, not justifying the benefits.

Analgesia duration with the 3-in-1 blockade in our study (13.1 \pm 2.47 hours) confirms other authors findings where analgesia duration was 15 hours with $0.25 \%$ bupivacaine ${ }^{1}, 14$ to 23 hours with $0.25 \%$ bupivacaine ${ }^{41,44,45}$ and 11 to 13 hours with $0.75 \%$ and $0.5 \%$ ropivacaine, respectively.

In Winnie's classical proposal, $20 \mathrm{ml}$ of anesthetic solution were used for 3-in-1 blockade. Our study used a higher volume aiming at decreasing the incidence of failures as suggested by other authors ${ }^{19,20}$. Our failure rate was similar to other studies were it has been $7 \%$ to $20 \%{ }^{1,41,45,46}$.

Different anesthetics may be used for 3 -in- 1 blockade, such as $0.75 \%$ prilocaine ${ }^{11}, 2 \%$ mepivacaine ${ }^{44}, 1 \%$ lidocaine ${ }^{11}$, $3 \%$ chlorprocaine ${ }^{11}, 0.25 \%$ and $0.5 \%$ bupivacaine $16,23,40,41,44,45$ and $0.5 \%$ or $0.75 \%$ ropivacaine $44,45,47$.

Ropivacaine was our drug of choice for its pharmacologic profile, such as long duration and low cardiotoxicity ${ }^{46,48}$. Many methods are described for 3-in-1 blockade, paresthesia seeking such as ${ }^{5}$, loss of resistance ${ }^{17}$ or with a peripheral nerve stimulators ${ }^{1,19,37,41,49,50}$. The last one has been the technique of choice for nerve location because, provided amperage and voltage limits are respected and if applied to mixed nerve ${ }^{51}$, it does not cause mechanical injury. This is why it was adopted in this study.

Prolonged motor block in group 3 -in-1 patients $(9.9 \pm 3.54$ hours) was a limiting factor to the technique. Thus, new studies are needed using lower anesthetic concentration to confirm the analgesic benefits of the technique with decreased motor block.

The advantage of lumbar plexus blockade by the 3-in-1 technique is the low incidence of complications. There are, however, reports on femoral neuropathies ${ }^{53}$ and hematoma-induced femoral nerve compressive syndrome ${ }^{53}$. Other reported complications are arterial puncture, intravascular local anesthetic injection, epidural anesthesia, infection at puncture site, nerve trauma, and regressive leg pain ${ }^{54-56}$. The low incidence of complications may be explained by decreased systemic local anesthetic absorption ${ }^{37}$. Our study confirms such observations by the lack of complications in the blockade groups.

The 3-in-1 technique is easy to perform, with low risk and short onset for pain relief. Our study has shown a favorable risk/benefit ratio among loco-regional techniques as compared to spinal morphine for postoperative analgesia in patients submitted to the procedures evaluated herein. The need for postoperative analgesics was $60 \%$ in group LPB and 
$75 \%$ in group $\mathrm{M}$, differently from Edkin et al. ${ }^{57}$ who reported $92 \%$ of patients not needing analgesia during the first 24 postoperative hours. This difference was probably due to the fact that postoperative analgesia was based on VAS and was administered whenever the score was 4 or above, aiming at patients' comfort and not waiting for their spontaneous request.

Spinal morphine has a higher incidence of side-effects, such as nausea, vomiting and pruritus ${ }^{58}$, as seen in our study. The lack of such effects in the 3-in-1 group suggests a major advantage for postoperative analgesia, added to its pasy performance in the supine position, with comfort to patients with manipulation restrictions, such as those with trans-skeletal traction, in addition to aiming at specific analgesia in the surgical site.

\section{REFERÊNCIAS - REFERENCES}

01. Imbelloni LE - Bloqueio 3 em 1 para analgesia pós- operatória. Rev Bras Anestesiol, 1999;49:412.

02. Tarkkila $P$, Tuominen M, Huhtala $\mathrm{J}$ et al - Comparison of intrathecal morphine and continuous femoral 3-in-1 block for pain after major knee surgery under spinal anaesthesia. Eur $\mathrm{J}$ Anaesth, 1998;15:6-9.

03. Kalso E - Effects of intrathecal morphine, injected with bupivacaine, on pain after orthopaedic surgery. $\mathrm{Br} \mathrm{J}$ Anaesth, 1983;55:415-422.

04. Bengtsson M, Löfström JB, Merits H - Postoperative pain relief with intrathecal morphine after major hip surgery. Reg Anesth, 1983;8:138-143.

05. Tarkkila P, Törn K, Tuominen $\mathrm{M}$ et al - Premedication with promethazine and transdermal scopolamine reduces the incidence of nausea and vomiting after intrathecal morphine. Acta Anaesthesiol Scand, 1995;39:983-986.

06. Singelyn FJ, Deyaert M, Jorist D et al - Effects of intravenous patient-controlled analgesia with morphine, continuous epidural analgesia, and continuous three-in-one block on postoperative pain and knee rehabilitation after unilateral total knee arthroplasty. Anesth Analg, 1998;87: 88-92.

07. Capdevila X, Barthelet $Y$, Biboulet $P$ et al - Effects of perioperative analgesic technique on the surgical outcome and duration of rehabilitation after major knee surgery. Anesthesiology, 1999;91:8-15.

08. Enneking FK, Wedel DJ - The art and science of peripheral nerve blocks. Anesth Analg, 2000;90:1-2.

09. Winnie AP, Ramamurthy S, Durrani Z - The inguinal paravascular technique of lumbar plexus anesthesia: the $3 \mathrm{em} 1$ block. Anesth Analg, 1973;52:989-996.

10. David LB - Atlas of Regional Anesthesia, $2^{\text {nd }} E d$, Philadelphia, Saunders Company, 1999;103-110.

11. Dalens B - Bloqueios do Plexo Lombar e de seus Ramos Proximais, em: Dalens B - Anestesia Locoregional do Recém-nascido ao Adulto, $1^{\text {a }} \mathrm{Ed}$, Rio de Janeiro, Editora Revinter, 1999;325-351.

12. Lonsdale M - 3-in-1 block: confirmation of Winnie's anatomical hypothesis. Anesth Analg, 1988;67:601-602.

13. Oliva Filho AL - Bloqueios de Nervos Periféricos, em: Manica JT - Anestesiologia Principios e Técnicas, $2^{\mathrm{a}}$ Ed, Porto Alegre, Editora Artes Médicas, 1997;379-380.
14. Misra U, Pridie AK, McClymont $C$ et al - Plasma concentrations of bupivacaine following combined sciatic and femoral 3 in 1 nerve blocks in open knee surgery. $\mathrm{Br} \mathrm{J}$ Anaesth, 1991;66: 310-313.

15. Patel NJ, Flashburg MH, Pashin S et al - A regional anesthetic technique compared to general anesthesia for outpatient knee arthroscopy. Anesth Analg, 1986;65: 186-187.

16. Tierney E, Lewis G, Hurtig JB et al - Femoral nerve block with bupivacaine $0.25 \%$ for post-operative analgesia after open knee surgery. Can J Anaesth, 1987;34:455-458.

17. Serpel MG, Millar FA, Thomson MF - Comparison of lumbar plexus block versus conventional opioid analgesia after total knee replacement. Anaesthesia, 1991;46: 275-277.

18. Schultz P, Anker-Moller E, Dahi JB et al - Postoperative pain treatment after open knee surgery: continuos lumbar plexus block with bupivacaine versus epidural morphine. Reg Anesth, 1991;16:34-37.

19. Tondare AS, Nadkarni AV - Femoral nerve block for fractured shaft of femur. Can Anaesth Soc J, 1982;29: 270-271.

20. Hood G, Edbrooke DL, Gerrish SP et al - Postoperative analgesia after triple nerve block for fractured neck of femur. Anaesthesia, 1991;46:138-140.

21. Imbelloni LE, Gouveia MA - Analgesia pós-operatória com bloqueio do plexo lombar: comparação entre as técnicas com cateter e com estimulação do nervo femoral. Rev Bras Anestesiol, 2001;51:28-36.

22. Imbelloni LE, Beato L, Gouveia MA - Bloqueio $3 \mathrm{em} 1$ para analgesia pós-operatória em cirurgias ortopédicas de grande porte. Rev Bras Anestesiol, 2000;50(Supl 25): CBA029A

23. Imbelloni LE - Bloqueio 3 em 1 com bupivacaína a $0,25 \%$ para analgesia pós-operatória em cirurgias ortopédicas. Rev Bras Anestesiol, 2000;50:221-224.

24. Fournier R, Van Gessel E, Gaggero G et al - Postoperative analgesia with 3 in 1 femoral nerve block after prosthetic hip surgery. Can J Anaesth, 1998;45:34-38.

25. Madej TH, Ellis FR, Halsali PJ - Evaluation of 3 in 1 lumbar plexus block in patients having muscle biopsy. $\mathrm{Br} \mathrm{J}$ Anaesth, 1989;62:515-517.

26. Ritter JW - Femoral nerve "sheath" for inguinal paravascular lumbar plexus blocks is not found in human cadavers. J Clin Anesth, 1995; 7:470-473.

27. Dalens B, Vanneuville G, Tanguy A - Comparison of the fascia iliac compartment block with the 3 -in-1 block in children. Anesth Analg, 1989;69:705-713.

28. Lang SA, Yip RW, Chang PC et al - The femoral 3-in-1 block revised. J Clin Anesth, 1993;5:292-296.

29. Edwards ND, Wright EM - Continuous low-dose 3-in-1 nerve blockade for postoperative pain relief after total knee replacement. Anesth Analg, 1992;75:265-267.

30. Parkinson SK, Mueller JB, Little WL et al - Extent of blockade with various approaches to the lumbar plexus. Anesth Analg, 1989;68:243-248.

31. Atanassoff PG, Weiss BM, Brull SJ et al - Electromyography comparison of obturator nerve block to three-in-one block. Anesth Analg, 1995;81:529-533.

32. Capdevila X, Biboulet P, Morau D et al - Continuous three-in-one block for postoperative pain after lower limb orthopedic surgery: where do the catheters go? Anesth Analg, 2002;94:1001-1006.

33. Huskisson EC - Measurement of pain. Lancet, 1974;2: 1127-1131.

34. Kremer E, Atkinson JH - Measurement of pain: patient preference does not confound pain measurement. Pain, 1981;10: 241-248.

35. Boogaerts JG, Vanacker E, Seidel L et al - Assessment of postoperative nausea using a visual analogue scale. Acta Anaesthesiol Scand, 2000;44:470-474.

Revista Brasileira de Anestesiologia Vol. 53, № 2, Março - Abril, 2003 
36. Cousins MJ, Mather LE - Intrathecal and epidural administration of opioids. Anesthesiology, 1984;61:276-310.

37. Dahl JB, Anker-Moller E, Spansberg N et al. Continuous lumbar plexus block after arthroplasty. Anaesthesia, 1988;43:989-996.

38. Hirst GC, Lang SA, Dust WN et al - Femoral nerve block: single injection versus continuous infusion for total knee arthroplasty. Reg Anesth, 1996;21:292-297.

39. Etches RC, Warriner CB, Badner et al - Continuous intravenous administration of ketorolac reduces pain and morphine consumption after total hip or knee arthroplasty. Anesth Analg, 1995;81:1175-1180

40. Tierney E, Lewis G, Hurtig JB et al - Femoral nerve block with bupivacaine $0.25 \%$ for postoperative analgesia after open knee surgery. Can J Anaesth, 1987;34:455-458

41. Mulroy MF, Larkin KL, Batra MS et al - Femoral nerve block with $0.25 \%$ or $0.5 \%$ bupivacaine improves postoperative analgesia following outpatient artroscopic anterior ligament repair. Reg Anesth Pain Med, 2001;26: 24-29.

42. Hugh A, Spencer L, Paul W et al - Peripheral nerve blocks improve analgesia after total knee replacement surgery. Anesth Analg, 1998;87:93-97.

43. Misra U, Pridie AK, McClymont $\mathrm{C}$ et al - Plasma concentrations of bupivacaine following combined sciatic and femoral 3 in 1 nerve blocks in open knee surgery. Br J Anaesth, 1991;66:310-313.

44. Fanelli G, Casati A, Beccaria P et al - A double-blind comparison of ropivacaine, bupivacaine and mepivacaine during sciatic and femoral nerve blockade. Anesth Analg, 1998;87:597-600.

45. Greengrass RA, Klein SM, Ercole FJ et al - Lumbar plexus and sciatic nerve block for knee arthroplasty: comparison of ropivacaine and bupivacaine. Can J Anaesth, 1998;45: 1094-1096.

46. Marhofer $P$ - Three in one block with ropivacaine: evaluation of sensory onset time and quality of sensory block. Anesth Analg, 2000;90:125-128.

47. Casati A, Cappeleni G - Regional anaesthesia for outpatient knee arthroscopy: a randomized clinical comparison of two different anesthetic techniques. Acta Anaesthesiol Scand, 2000;44:543-547.

48. Reiz S, Haggmark S, Johansson G et al - Cardiotoxicity of ropivacaine - a new amide local anaesthetic agent. Acta Anaesthesiol Scand, 1989;33:93-98.

49. Seeberger MD, Urwyler A - Paravascular lumbar plexus block: block extension after femoral nerve stimulation and injection of 20 vs $40 \mathrm{ml}$ mepivacaine $10 \mathrm{mg} / \mathrm{ml}$. Acta Anaesthesiol Scand, 1995;39:769-773.

50. Allen JG - Postoperative analgesia following total knee arthroplasty. A study comparing spinal anesthesia and combined sciatic-femoral 3 in 1 block. Reg Anesth Pain Med, 1998; 23:142-146.

51. Yassuda H, Sakata RK - Bloqueio de Nervos Periféricos, em: Yamashita AM, Takaoka F, Auler Jr JOC et al - Anestesiologia,

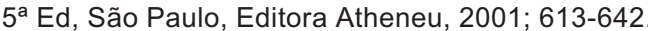

52. Uhrbrand $A B$, Jensen TT - latrogen $n$. femoralis neuropathy after blockade of plexus lumbalis (3-i-1 block). Ugeskr Laeger, 1988; 150:428-429.

53. Joehr M - Spaete komplikation der kontinuierlichen blocade des n. femoralis. Teg Anaesth, 1987;10:37-38.
54. Lynch J, Trojan S, Arhelger S et al - Intermittent femoral nerve blockade for anterior cruciate ligament repair. Use of a catheter technique in 208 patients. Acta Anaesthesiol Belg, 1991;42: 207-212.

55. Lalourcey L, Nouvelon E, Cuvillon P et al - Incidence des complications des blocs 3 en 1 pour analgésie continue chez 125 patients. Ann FR Anesth Réanim, 1998;17:822.

56. Singelyn FJ, Contreras V, Gouverneur JM - Epidural anesthesia complicating continuous 3 -in-1 lumbar plexus blockade. Anesthesiology, 1995;83:217-220.

57. Edkin BS - Femoral nerve block as an alternative to parenteral narcotics for pain control after anterior cruciate ligament reconstruction. Artroscopy, 1995;11:404-409.

58. Kehlet H, Dahl JB - The value of 'multimodal' or 'balanced analgesia' in postoperative pain treatment. Anesth Analg, 1993;77:1048-1056.

\section{RESUMEN}

Fonseca NM, Ruzi RA, Ferreira FX, Arruda FM - Analgesia Pós-Operatoria en Cirugía Ortopédica: Estudio Comparativo entre el Bloqueo del Plexo Lombar por Vía Perivascular Inguinal (3 en 1) con Ropivacaína y la Analgesia Subaracnóidea con Morfina

Justificativa y Objetivos - El bloqueo del plexo lumbar por el acceso perivascular inguinal, llamado de bloqueo 3 en 1, ha sido utilizado para analgesia pós-operatoria. El objetivo de este estudio fue comparar la analgesia pós-operatoria del bloqueo 3 en 1 a la de la morfina subaracnóidea en pacientes sometidos a cirugías ortopédicas en miembro inferior (MI).

Método - Fueron estudiados 40 pacientes escalados para cirugía ortopédica de MI, de ambos sexos, estado físico ASA I y II, con edades entre 15 y 75 años, distribuidos en 2 grupos ( $M$ y $B P L)$. Fue realizada anestesia subaracnóidea en todos los pacientes, en $L_{3}-L_{4} O L_{4}-L_{5}$, con 20 mg de bupivacaína isobárica a $0,5 \%$. En el grupo $M(n=20)$ fue asociado $50 \mu$ de morfina al anestésico local. En el grupo BPL $(n=20)$ fue realizado el bloqueo 3 en 1 al término de la cirugía, utilizando 200 mg de ropivacaína a $0,5 \%$. Se evaluó la analgesia y la intensidad del dolor a las 4, 8, 12, 14, 16, 20 y 24 horas después del término de la cirugía, el nivel del bloqueo subaracnóideo, el tiempo quirúrgico y las complicaciones.

Resultados - La duración de la analgesia en el grupo BPL fue de $13,1 \pm 2,47$, en cuanto en el grupo $M$ todos los pacientes referían dolor y ausencia de bloqueo motor en el primero instante evaluado (4 horas). Hubo falla del bloqueo de uno de los 3 nervios en 3 pacientes. La incidencia de náusea y prurito fue significativamente mayor en el grupo $M$. Cuanto a la retención urinaria, no hubo diferencia significante entre los grupos. No hubo depresión respiratoria, hipotensión arterial o bradicardia. La analgesia pós-operatoria fue mas efectiva en el grupo BPL, comparada al grupo M a las 4, 8, 12,14 y 16 horas. A las 20 y 24 horas no hubo diferencia significante entre los grupos.

Conclusiones - La analgesia pós-operatoria proporcionada por el bloqueo 3 en 1 presentó efectos colaterales inferiores a la morfina subaracnóidea con tiempo de analgesia semejante. 